\author{
Pía Ibarra Yáñez \\ Universidad de Santiago de Chile \\ pia.ibarra.y@usach.cl \\ Camila Amoyao Bravo \\ Universidad de Santiago de Chile \\ camila.amoyao@usach.cl \\ Eduardo Román Álvarez \\ Universidad de Santiago de Chile \\ eduardo.roman@usach.cl
}

\title{
Análisis de la cobertura de la prensa digital chilena durante la discusión legislativa de la Ley de Identidad de Género en 2018
}

\author{
Analysis of the coverage of the Chilean digital press during the \\ legislative discussion of the Gender Identity Act in 2018
}

\section{Resumen}

Este artículo analiza la cobertura de los medios digitales sobre la discusión de la Ley de Identidad de Género en Chile en septiembre de 2018. El estudio fue abordado durante tres días antes de la votación, el día del debate en el Congreso y tres días después de aprobada. Se busca mostrar de qué forma los medios de comunicación digital construyeron realidad, siendo el principal objetivo analizar la cobertura de la prensa digital durante ese episodio, sosteniendo como hipótesis que la prensa realizó una cobertura poco acuciosa, principalmente por el manejo de las fuentes. Se analizaron datos obtenidos a través de la plataforma NewsReport, arrojando la distribución de los medios con mayor cobertura de la noticia, los actores políticos más relevantes, las palabras claves y fuentes de las noticias, y el marco temporal de mediatización. Se concluye que la prensa tendió a la neutralidad pero con un manejo de fuentes precario.

Palabras clave: Ley de Identidad de Género, Chile, Construcción de la realidad, Prensa.

\footnotetext{
Abstract

This article discusses digital media coverage of the discussion of the Gender Identity Act in Chile in September 2018. The study was approached for three days before the vote, on the day of the congressional debate and three days after it was approved. It was raised to know how the digital
} 
Pía Ibarra Yáñez - Camila Amoyao Bravo - Eduardo Román Álvarez

media builds reality, being the main objective to analyze the coverage of digital press during that episode, arguing as hypothesis that the press made an unquesqueous coverage, mainly by the management of sources. Data obtained through the NewsReport platform were analyzed, yielding media distribution with the highest news coverage, the most relevant political actors, keywords and news sources, and the time frame of mediatization. Concluding that the press tended to neutrality, but with a precarious source management.

Keywords: Gender Identity Act, Chile, Construction of reality, Press, Gender.

\section{Introducción}

En plenos años setenta, comenzó a emerger en occidente el movimiento por la "Liberación Gay". La lucha de la llamada comunidad LGBTI (Lesbianas, Gays, Bisexuales, Transgénero/Transexuales e Intersexuales) estaba basada en líneas generales, en el derecho al reconocimiento de sus orientaciones sexuales. En aquel entonces ni se vislumbraba la posibilidad de reivindicar mayores derechos (Weeks, 2012).

En el plano latinoamericano, con la caída de las dictaduras que marcaron los años 70s y 80s y que estuvieron marcados por la restricción de las libertades civiles, comenzó un proceso de apertura en lo que respecta a la promoción de los derechos de estas personas. Esto empezó a darse en la discusión de legislaciones relativas a la "anti-discriminación", derechos en salud y en lo social (Díez, 2013).

En ese sentido, empezaron a gestarse esfuerzos internacionales por normar aspectos específicos de las vidas de personas con orientaciones sexuales e identidades de género diversas. Un paso importante fue la producción de los "Principios de Yogyakarta sobre la Aplicación de la Legislación de los Derechos Humanos en relación con la Orientación Sexual y la Identidad de Género", los que, a pesar de no configurar un tratado de índole internacional, sí logró fijar lineamientos legales para intentar frenar el avance de la violencia y discriminación hacia personas LGBTI (Alto Comisionado de las Naciones Unidas para los Refugiados [ACNUR], 2014).

Durante el último tiempo los problemas de personas transgénero lograron tener más visibilidad, lo que ha suscitado interés de la opinión pública, puesto que han salido a la luz las problemáticas que atraviesan. Una de las mayores dificultades que han enfrentado tiene que ver 
Análisis de la cobertura de la prensa digital chilena durante la discusión legislativa de la Ley de Identidad de Género en 2018

Pía Ibarra Yáñez - Camila Amoyao Bravo - Eduardo Román Álvarez

con el reconocimiento legal de su identidad de género, o la carencia de mecanismos que acceder a herramientas para cambiar el sexo estipulado en los documentos de identificación (Rangel, 2016).

Ese sería uno de los mayores inconvenientes que atraviesan, puesto que el desconocimiento de la existencia de estos sujetos como individuos impide que puedan disfrutar de forma igualitaria al resto de la población, de una mayor protección de sus derechos (Programa de las Naciones Unidas Para el Desarrollo [PNUD], 2014).

A nivel latinoamericano, en Argentina la Ley de Identidad de Género (Ley $\mathrm{N}^{\circ}$ 26.743) se reglamentó en 2012. Esta consiste en que las personas mayores de edad, si lo solicitan, pueden modificar "su nombre y sexo en Documento Nacional de Identidad (DNI) sin necesidad de autorización judicial o administrativa, ni de ningún otro tipo de informe o diagnóstico biomédico". (Braz, 2019: 124). El proceso para los menores de edad debe "ser acompañado por su representante legal y un abogado. La referida ley prevé cobertura en todos los sectores del sistema de salud, solicitando sólo la firma de un consentimiento informado". (Braz, 2019: 124). La consecuencia mediática de la ley en Argentina fue intensa entre los conservadores y los activistas, según lo mencionó Braz (2019), ya que su proceso duró “cerca de tres años después de su aprobación en 2015, lo que implicó no sólo espera, sino que demandó la movilización por parte de activistas". (p. 124).

La ley de identidad de género (Ley $\mathrm{N}^{\circ}$ 807) que fue aprobada el 2016 en Bolivia tiene un contenido similar a la ley de Argentina. (Quiteria, 2017). Por otro lado, respecto a la comunicación con perspectiva de género, es considerado uno de los países con políticas avanzadas con "la Ley General de Telecomunicaciones y la Ley 348 para una vida libre de violencia integran el derecho a la comunicación de todas y todos los ciudadanos de la nación" que tiene el propósito de desmoronar los estereotipos patriarcales e incorporar a las diversidades de toda índole. (Ochoa Chaves, Castro Camacho, \& Cubero Hernández, 2017: 61).

En relación a Uruguay, fue un país pionero en legislar el derecho a la identidad de género (Ley $\mathrm{N}^{\circ} 18.620$ ) en el 2009, normativa que consiste en el cambio de nombre y sexo en la cédula de identidad donde "se requiere de la presentación de un informe por parte de un equipo multidisciplinario" (Quiteria, 2017). Por el lado de la cobertura noticiosa en temáticas de género, poseen leyes intermedias, las que incorporan "medidas de prohibición de contenidos que 
Análisis de la cobertura de la prensa digital chilena durante la discusión legislativa de la Ley de Identidad de Género en 2018

Pía Ibarra Yáñez - Camila Amoyao Bravo - Eduardo Román Álvarez

promueven la discriminación por género y -en algunos casos- la definición de la violencia mediática y simbólica”. (Ochoa Chaves, Castro Camacho, \& Cubero Hernández, 2017: 61).

Con respecto a Colombia, el cambio de nombre y sexo en la documentación de las personas trans, se legisló en 2015 por medio del decreto 1227. Mediáticamente, las noticias abarcadas en cuanto a la población LGBTI "muestran la intolerancia de la sociedad y cómo operan los imaginarios en estos medios de comunicación" bajo ciertos perjuicios y una mirada moralizadora, ya que en el país hay sectores conservadores radicales que provienen de Iglesias y un gobierno novato en el tema. (Pájaro, 2016: 170).

En el 2017, Costa Rica adoptó la "Ley de Reconocimiento de los Derechos a la Identidad de Género e Igualdad" (Ley N ${ }^{\circ}$ 19.841), pero a pesar de este importante avance en el reconocimiento de identidad de personas trans y la definición de los términos (género, identidad sexual e imagen de la persona), Costa Rica "posee una legislación en comunicación "del siglo pasado", por lo que la vinculación con género está muy poco presente". (Ochoa Chaves, Castro Camacho, \& Cubero Hernández, 2017: 65).

Luego de cinco años en el Congreso, el 4 de septiembre de 2018 se aprobó en la legislatura chilena la Ley de Identidad de Género (LIG), la que contempló el cambio de nombre y sexo registral de personas trans mayores de 14 años. Este paso fue catalogado como histórico por miembros de la diversidad sexual, pero un tanto insatisfactorio debido a la no inclusión de menores de 14 años en la ley, pues significaría una vulneración hacia sus derechos.

Por otro lado, el descontento se notó entre miembros de partidos de derecha, grupos religiosos, y quienes consideraron que esta ley no debiera tener cabida, puesto que atentaría contra los principios morales que pregonan.

A partir de ello, esta investigación se propuso analizar el comportamiento medial que se dio en Chile sobre el tema, su cobertura y abordaje. Debido a que la diversidad sexual se ha utilizado en variadas ocasiones en los medios de comunicación como insumo para el humor y la ridiculización, y aquellas "lógicas de producción periodística en algunos medios refuerzan la discriminación, y aún más grave es la caricaturización, la que contraviene derechos y demandas históricas levantadas por los movimientos de diversidad sexual". (Fries, 2016: 39). 
Pía Ibarra Yáñez - Camila Amoyao Bravo - Eduardo Román Álvarez

El marco temporal de la investigación se encuentra regido desde tres días antes de aprobada la ley, cuando el 10 de septiembre "la Corte Suprema, dio su parecer a petición de la presidenta de la Comisión Mixta" (Iguales, s.f.).

Además, contempla el día de la votación de la ley (12 de septiembre), cuando se aprobó la regulación de los casos de los mayores de 14 años, con 95 votos a favor y 46 en contra, sin ninguna abstención, y, por otra parte, se rechazó la regulación de los casos a menores de 14 años, con 73 votos a favor y 66 en contra, existiendo solo una abstención. "Luego de esto, el proyecto fue despachado. El mismo 12 de septiembre la Cámara de Diputados envió el oficio a la Cámara del Senado" (Iguales, s.f.). Asimismo, se abordaron los tres días posteriores a la aprobación. En el trayecto de su tramitación, más tarde, "el 28 de noviembre, es promulgada por el Presidente, Sebastián Piñera. Y, finalmente, el 10 de diciembre de 2018, fue publicada en el Diario Oficial la ley $21.120 "$ (Iguales, s.f.).

La pertinencia del tema radica en que significa una problemática universal en el siglo XXI, que preocupa a todas las disciplinas de forma transversal, y de manera particular al estudio de las comunicaciones, pues en la era de la globalización y la sociedad de los dispositivos tecnológicos que facilitan enormes flujos de información, estos consiguen instalar en la sociedad discursos que representan a los cuerpos no hegemónicos de la forma que cada línea editorial crea conveniente, lo que se vuelve aún más importante en el caso chileno en donde existen niveles de concentración escandalosos.

La pregunta de investigación de este estudio es: ¿De qué forma los medios de comunicación digital en Chile construyeron realidad en el periodo de discusión legislativa de la Ley de Identidad de Género aprobada en 2018? En tanto que el objetivo general es analizar la cobertura de los medios digitales chilenos durante la discusión de la LIG. Los objetivos específicos persiguen comparar el tratamiento de fuentes en los distintos medios, y examinar los flujos de información respecto al tema en la prensa digital chilena.

Esta investigación se inició con los datos obtenidos en un trabajo de investigación de la cátedra de Teoría de la Comunicación y ha sido complementado en trabajos de asignaturas posteriores en la Universidad de Santiago de Chile. 
Análisis de la cobertura de la prensa digital chilena durante la discusión legislativa de la Ley de Identidad de Género en 2018

Pía Ibarra Yáñez - Camila Amoyao Bravo - Eduardo Román Álvarez

\section{Marco Teórico}

Hace un siglo, los medios de comunicación se instalaban en el cotidiano social con una fuerza inusitada. A caballo con las nuevas tecnologías, los periódicos y las radios se asumían como "cuarto poder" gracias a su enorme influencia sobre las masas. Con sus nuevos productos - las noticias, la actualidad, el mundo acotado — asumidos en plenitud por las personas, se convirtieron en los constructores, quizás inconscientes, de la realidad. Y no sólo en la presentación de hechos objetivados, sino en la semantización misma de los acontecimientos.

Paradójicamente, en los inicios del siglo XXI, las nuevas tecnologías generaron un espacio competitivo - redes sociales y medios alternativos-, que ha puesto en duda las competencias semantizadoras de los medios de comunicación masiva. Y no se trata solo de una adaptación a otros códigos y soportes, sino a su capacidad para integrar temas y enfoques ideológicos diferentes a sus rituales profesionales y menos aún a sus relatos.

De esta manera, los medios chilenos tuvieron que cubrir un debate de fondo en la sociedad chilena: La Ley de Identidad de Género (LIG), que refiere al cambio de nombre y sexo registral de personas trans mayores de 14 años. Como el tema, en términos religiosos, opone a los sectores conservadores con los más liberales en términos de aceptación de la diversidad sexual, también los públicos optaron por informarse en los medios tradicionales o alternativos, según sus propias preferencias y valoraciones.

Como este artículo refiere, de modo comparado, a la información que difundieron los medios de comunicación sobre la implementación de la Ley de Identidad de Género, cabe revisar, precisamente, cómo operan los medios en su rol de semantizadores de la realidad.

En nuestra vida cotidiana, la conexión con las informaciones de la televisión o de otros medios tecnológicos constituye un rito que nos vincula con el mundo, pero estos medios no ofrecen los hechos, pues no hay modo de reproducirlos, sino un relato mediatizador presentado a modo de mosaico. Gomiz explica que el periodismo es una actividad profesional de mediación, el fruto de una organización que se dedica precisamente a interpretar la realidad social y a mediar entre los que hacen de productores de espectáculo mundano y la multitud que cumple funciones de público (Gomiz, 1991: 128). 
Pía Ibarra Yáñez - Camila Amoyao Bravo - Eduardo Román Álvarez

En efecto, la verdad "se presentaría lenta, difusa y aburrida. Por este motivo, el Periodismo presenta una versión inventada, dramatizada, sugestiva, que escoge lo más interesante (..) y lo retoca lo suficiente para ajustarlo a las exigencias de tiempo y espacio" (Gomiz, 1991: 129). En este sentido, la función de los medios es "proveernos de un presente social continuo, bastante nuevo para que nos impresione y bastante viejo para que podamos conocerlo y comentarlo, de manera de dominarlo" (Gomiz, 1991: 130).

Los hechos construidos por los medios repercuten en comentarios y consecuencias, que forman parte de una unidad: repercusiones que se suman para cuantificar el impacto. No todas las repercusiones de noticias en la sociedad quedan registradas en los medios, pero sí suficientes para apreciar su influencia en la sociedad y evaluar su éxito. Al destacar aquellos hechos con más repercusiones potenciales, los medios proyectan sobre el porvenir de la sociedad y en cada entrevista seleccionada se destacan las preferencias de cada persona, donde prima una opinión sobre otra: "Las preferencias de cada uno tienden a despertar o intensificar el interés por las noticias. Las opiniones recogidas por los medios también dan pauta a la producción de hechos por los interesados en captar las preferencias del público" (Gomiz, 1991: 142).

Teun van Dijk indica que la noticia constituye un discurso público. En oposición a las cartas personales o las publicaciones especializadas, sus lectores son grupos grandes, a veces definidos por alianzas políticas o ideológicas similares, pero normalmente indeferenciados en un nivel más personal. Lo anterior significa que, socialmente, una considerable cantidad de conocimientos, creencias, normas y valores deben ser reafirmados. Sin una información que pueda darse por sentada, la noticia no sería inteligible, por eso su propósito es regularmente actualizarla. Por eso, en el estilo debe llevar los indicadores de estas presuposiciones compartidas y del modo más sencillo:

"Los periodistas tienden a ser reacios a aceptar conocimientos de cualquier investigación académica en su arte. La edición y corrección de estilo, entonces, se basa en su mayor parte, en conocimientos intuitivos, la rutina profesional y 
Pía Ibarra Yáñez - Camila Amoyao Bravo - Eduardo Román Álvarez

el sentido común, lo cual por supuesto es a menudo suficiente para el cumplimiento eficaz de las tareas rutinarias cotidianas". (Van Dijk, 1990: 86).

La retórica del discurso tiene que ver con el modo como decimos las cosas, pero como el estilo periodístico se halla muy limitado por los factores contextuales y la naturaleza formal de las noticias, el uso de estructuras retóricas depende de los efectos buscados por la comunicación. Desde el punto de vista ideológico, la noticia promueve implícitamente las creencias y opiniones dominantes de grupos de élite en la sociedad, difundiendo una serie de informaciones que delimitan, en cierta medida, el horizonte cognitivo del público.

Los medios de comunicación se presentan como constructores de la realidad social, ya que se autodefinen como transmisores de la "actualidad". Rodrigo Alsina aclara que "si conceptuamos la "actualidad" no ya como todo aquello que sucede en el mundo y que puede ser transformado en noticia, sino únicamente los acontecimientos a los que tienen acceso los mass media, aun así, la "actualidad", transmitida en forma de noticia, no es más que una pequeña parte de estos acontecimientos" (Rodrigo Alsina, 1993: 48). En este sentido, cabe reiterar la base de la teoría de la construcción del temario (Agenda Setting), que apunta claramente a que es muy posible que los medios de comunicación no tengan el poder de trasmitirle a la gente cómo deben actuar, pero sí consiguen imponer al público cómo interpretar los acontecimientos.

La función de los medios es interpretar sucesivamente la realidad social y esto es posible porque reduce la realidad a noticias, las noticias ampliadas a crónicas, las crónicas a reportajes, etc. Así, siempre está "formando una imagen cambiante de la realidad, de lo que se sirve el público para estar enterado, comentar e intervenir en ella y de que se sirven también los protagonistas de las acciones sociales para lo mismo" (Gomiz, 1991: 89). Es decir, lo que no pasa por los medios, no pasa en ninguna parte, no influye.

En la selección de noticias, los medios siguen dos principios: el de la universalidad, que se puede resumir con frase "nada de lo que pase puede quedar excluido de la posibilidad de convertirse en noticia, suceda donde suceda y lo haga quien lo haga, siempre que sea un hecho novedoso", y el de neutralidad, que se traduce en "las noticias no son buenas ni malas, son moralmente neutras" (Gomiz, 1991: 90). 
Pía Ibarra Yáñez - Camila Amoyao Bravo - Eduardo Román Álvarez

Los medios trabajan con abundancia de noticias, favorecidos por la enorme cantidad de fuentes interesadas, por la multiplicidad de sus recursos y por la tecnología emergente. Como el trabajo de selección no es en equipo y la captación y selección de noticias pasa generalmente por varias manos, la referencia -por lo menos implícita- a la hora de escoger será qué hecho es más noticia que otro.

El periodista que logra hacer llegar el hecho escogido a una audiencia, influye efectivamente. Desde la perspectiva política, influir en el público es reforzar el poder que se tiene, ya que la política consiste básicamente en una producción de hechos capaces de ser aceptados y difundidos.

Según Lorenzo Gomiz, el Periodismo puede considerarse un método de interpretación sucesiva de la realidad social y, como tal, establece supuestos:

- $\quad$ La realidad puede fragmentarse en períodos. El único que se intenta explicar es el actual (el aún no interpretado).

La realidad puede fragmentarse en unidades completas e independientes, posibles de interpretar en fragmentos breves y autónomos (noticias).

- La realidad interpretada debe poder asimilarse en tiempos variables por públicos heterogéneos.

- La realidad interpretada debe encajar en un espacio o tiempo dados (programación del medio).

- $\quad$ Los medios se valen de una serie de filtros o formas convencionales (géneros) que van de la información pura al comentario polémico, para que se capte la realidad.

En el mismo sentido, la interpretación de la realidad se realiza en tres niveles:

- Interpretación de hechos: componen el presente social como un mosaico de acontecimientos;

Interpretación de situaciones: presenta personajes y situaciones en un ámbito temático o lugar del mundo, complementando el nivel anterior (crónica y reportaje);

- Interpretación moral: analiza o juzga hechos y situaciones. Su función es esclarecer si son buenos o malos; convenientes o no; y proyectar esos juicios sobre las acciones 
Análisis de la cobertura de la prensa digital chilena durante la discusión legislativa de la Ley de Identidad de Género en 2018

Pía Ibarra Yáñez - Camila Amoyao Bravo - Eduardo Román Álvarez

necesarias para conseguir que el futuro sea mejor (comentario, editorial, artículo) (Gomiz, 1991: 122).

Desde una perspectiva dialéctica, Adoni y Mane plantean tres tipos de representaciones que generan los medios de comunicación, en el proceso de construcción social:

a) La realidad social objetiva. Es experimentada como el mundo objetivo que existe fuera del individuo. Esta realidad es aprehendida por los individuos como algo que no necesita verificación, enmarcando el sentido de los actos de existencia cotidianos.

b) La realidad social simbólica. Consiste en las distintas formas de expresión de la realidad objetiva, que pueden manifestarse en un caudal de diferentes sistemas de símbolos. Sin embargo, el individuo está capacitado para diferenciar las distintas esferas de realidad simbólicas.

c) La realidad social subjetiva. Tiene como imputs a las otras dos, es decir, el mundo objetivo y las representaciones simbólicas se han fundido en la realidad social subjetiva. Las construcciones individuales de la realidad se basan en las acciones y configuraciones personales de las personas. (Adoni y Mane, 1984: 188).

En el mismo sentido, Hall recoge y analiza tres funciones ideológicas de los medios:

a) Suministro y construcción selectiva del conocimiento social. El conocimiento social depende de la mediación periodística, puesto que los medios proporcionan información sobre grupos y clases, de forma que sus integrantes construyen una imagen de las cosas, significados, prácticas y valores, tanto de sí mismos como de otros grupos y clases. Por otro lado, suministran imágenes y representaciones sobre la totalidad social. Es decir que las piezas fragmentadas que se transmiten pueden ser captadas coherentemente como una totalidad.

b) Reflejar una pluralidad social. Los medios suministran un inventario de léxicos, estilos de vida e ideologías, para que así los distintos tipos de conocimiento social sean calificados, ordenados y asignados en sus contextos referenciales, dentro de los mapas de la realidad social. Sus calificaciones son evaluativas y normativas, pero no sólo sirven para saber más sobre el mundo sino para darle un sentido. 
Pía Ibarra Yáñez - Camila Amoyao Bravo - Eduardo Román Álvarez

c) Organizar, orquestar y unir lo que se ha representado y clasificado selectivamente, dentro de un orden reconocido. Este aspecto ideológico, clave en los medios, está constituido por la producción del consenso, y la construcción de la legitimidad del proceso de argumentación, intercambio, debate, consulta y especulación que sostienen dicho consenso. (Hall, 1981: 36).

Dentro del proceso de construcción social, la noticia se constituye en un tipo especial de "realidad". Por lo tanto, esta relación entre el medio y sus destinatarios está establecida por un contrato fiduciario social e históricamente definido:

"A los periodistas se les atribuye la competencia de recoger los acontecimientos y temas importantes y atribuirles un sentido. Este contrato se basa en unas actitudes epistémicas colectivas que se han ido forjando por la implantación del uso social de los medios de comunicación como transmisores de la realidad social de importancia pública. Los propios medios son los primeros que llevan a cabo una continua práctica de autolegitimación para reforzar este rol social". (Alsina, 1993: 167).

Ya sea en niveles directivos o dependientes, los periodistas se dedican a interpretar los hechos para el público. Por lo tanto, su función es semantizar la realidad. Una manera de hacerlo es simulando que los acontecimientos fluyen por sí mismos y la otra es asumiendo la responsabilidad social que implica participar en la construcción de la noticia. Lo anterior no implica que la única posibilidad sea manipular la información. Por el contrario, la conciencia del proceso permite la articulación de las informaciones sobre una base explícita de valores éticos. Por el contrario, la "ingenuidad" comunicacional avala una manipulación soterrada, que es mucho más peligrosa precisamente porque carece de controles sociales.

Semantizar la realidad es construir relaciones entre los códigos implícitos y también con su contexto, considerando que: 
Pía Ibarra Yáñez - Camila Amoyao Bravo - Eduardo Román Álvarez

"Los códigos periodísticos son afectados por el habla de los públicos, por los significados y formas de expresión literarias, por las terminologías en uso dentro de las actividades deportivas, sociales y culturales; por la propaganda comercial e ideológica y por los códigos de la jerga periodística que se forma en la dinámica de esta profesión”. (Rivadeneira, 1991: 93)".

En resumen, los medios no solo informaron sobre los trámites relativos a la Ley de Identidad Sexual, sino que expusieron los marcos interpretativos -ideológicos, religiosos y epistemológicos- en los cuales sus públicos pueden desplegar una semantización de los acontecimientos.

\section{Fundamentos metodológicos}

La presente investigación inició en septiembre de 2018, donde se comenzó a recopilar y analizar noticias sobre el tema, mientras que el análisis se extendió hasta noviembre de 2020.

Se utilizó la plataforma web de pago NewsReport, una base de datos que recoge y clasifica noticias digitales de todos los medios de diversos países. Cabe destacar que dicho sitio web dejó de existir.

Se realizó un libro de códigos en donde se le asignaron valores a las variables. Asimismo, se elaboró una ficha de clasificación y registro en donde se identificaron y seleccionaron los datos anotando el titular correspondiente en una planilla de cálculo. Posteriormente, se registraron los links, y luego se etiquetó la información utilizando una o más categorías contenidas en el/los texto/s recogido/s, incluyendo la revisión de elementos digitales adicionales al texto, como audios o vídeos adjuntos.

Las variables fueron: Temática (la materia principal de la que trate la noticia, en este caso, en relación a lo que los medios dicen o informan sobre la Ley de Identidad de Género. También las referencias que explican o desarrollan relatos conectados con este tópico. Se dividió este ítem en temáticas de Derechos, Vulneración de Derechos y Opinión); Características de la Noticia (se analizaron los Medios que entraban en el universo del estudio, el Formato 
Pía Ibarra Yáñez - Camila Amoyao Bravo - Eduardo Román Álvarez

Periodístico, el Tratamiento de la Noticia según una visión Favorable, Desfavorable o Neutral, y la Temporalidad); y el Trabajo de Fuentes (según Tipos de Fuentes, Actores Políticos y Número de Fuentes).

La muestra considerada para este estudio fueron los medios de prensa digital, más abajo mencionados. Los que no fueron incluidos se debió a que no abordaban la temática analizada (por ejemplo, "Heureka", periódico digital que se enfoca en ciencias y tecnología) o por no tener información escrita, sino solo multimedia.

El tiempo que abarcó el estudio fue desde el 1 al 7 de septiembre de 2018. Esto porque se consideró el tratamiento informativo que tuvo la noticia los 3 días anteriores a la discusión de la LIG en el Congreso, el día de la votación en el Senado chileno, y los 3 días posteriores.

Los tipos de información considerados fueron informativos, interpretativos y opinión. La muestra consideró los siguientes medios:

24Horas, AdnRadio, El Líbero, Bio-Bio Chile, Cooperativa, Diario Concepción, El Ciudadano, El Desconcierto, El Dínamo, El Mostrador, El Siglo, EMOL, La Cuarta, La Segunda, La Tercera, Publimetro, Radio Agricultura, Soy Chile, The Clinic

\section{Principales resultados y análisis:}

Figura 1. Distribución de la cobertura según cada medio

La categoría ilustra sobre el tratamiento informativo de la LIG que tuvieron los medios chilenos seleccionados. Encontrando que quienes más abordaron el tema fueron La Tercera, El Dínamo y Radio Agricultura.

Por otra parte, los medios que menos registraron la noticia son ADN, El Siglo, El Líbero y La Cuarta. 
Análisis de la cobertura de la prensa digital chilena durante la discusión legislativa de la Ley de Identidad de Género en 2018

Pía Ibarra Yáñez - Camila Amoyao Bravo - Eduardo Román Álvarez

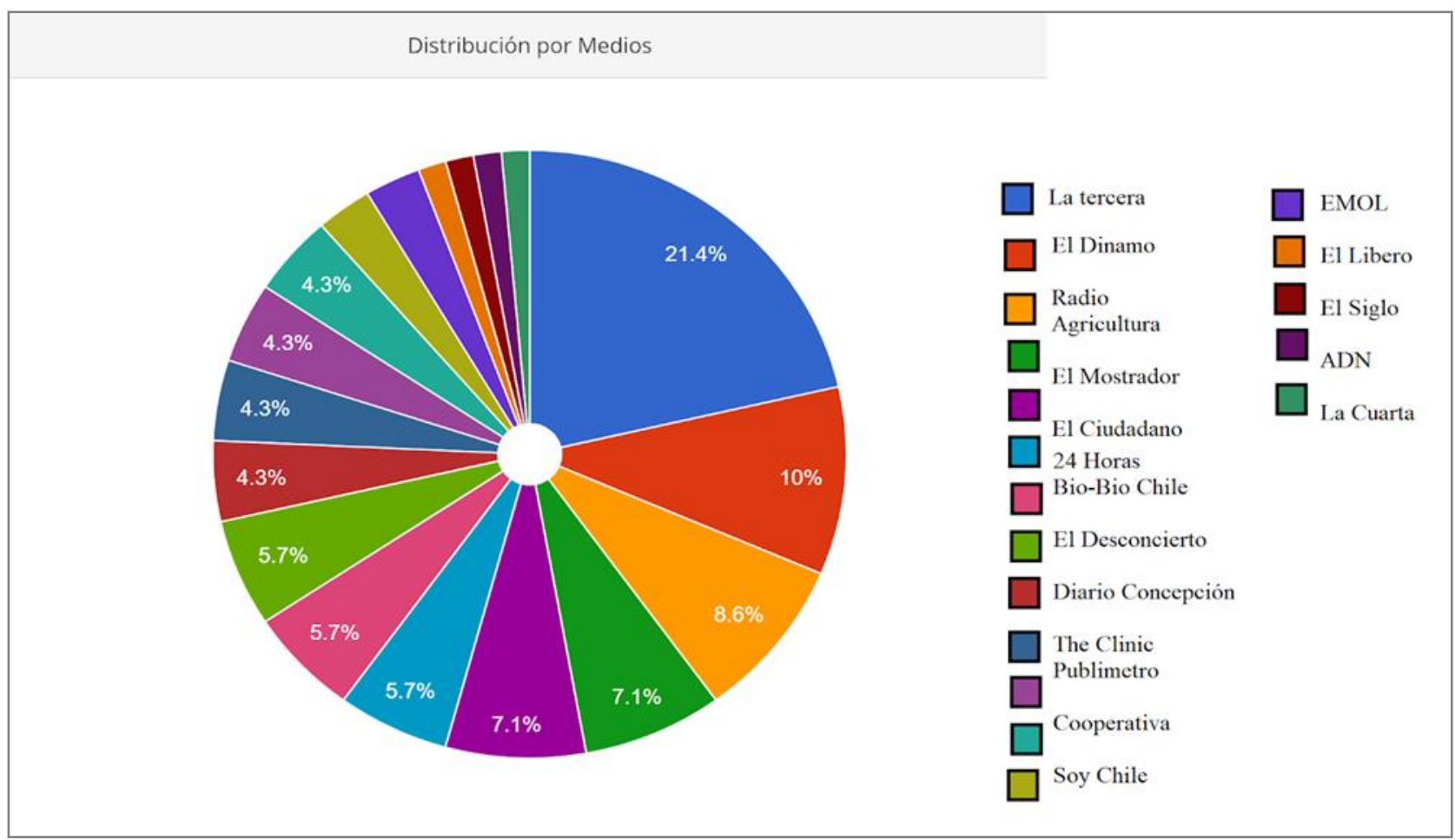

Figura 1. Distribución de la cobertura según cada medio. Fuente: Elaboración propia (2018).

Figura 2. Posicionamiento medial de agrupaciones de la sociedad civil y partidos políticos incidentes.

El gráfico lineal muestra comparativamente el posicionamiento, medido a partir de la cantidad de menciones en la prensa, que obtuvieron en los medios las agrupaciones incidentes (Frente Amplio, UDI, y Movilh). Esto da cuenta de que el Movilh (agrupación de la sociedad civil), siendo uno de los principales impulsores de la LIG, en general tuvo una muy baja cobertura en los medios. Aquello en contraposición a los partidos y conglomerados políticos, quienes fueron los más nombrados. 
Análisis de la cobertura de la prensa digital chilena durante la discusión legislativa de la Ley de Identidad de Género en 2018

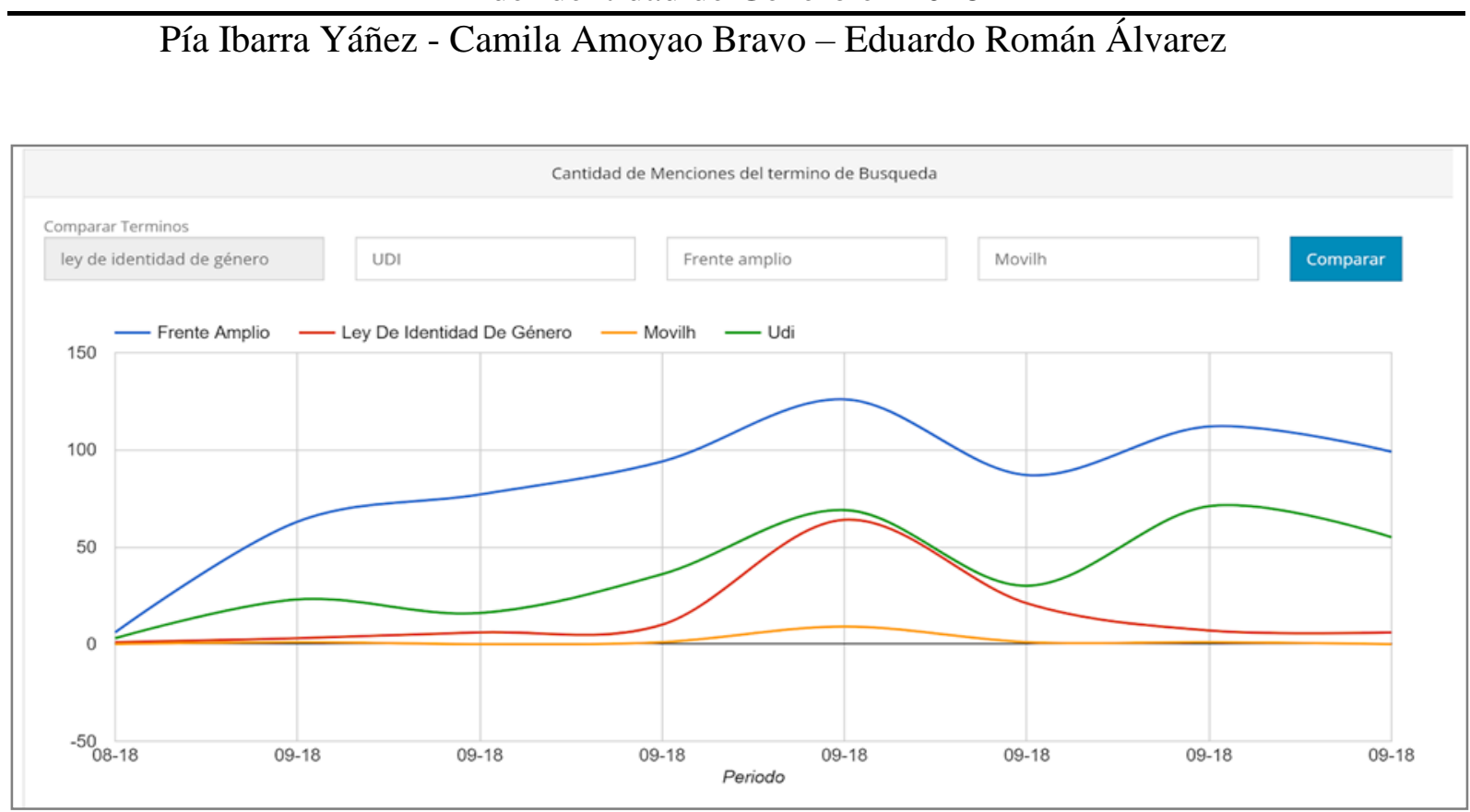

Figura 2. Posicionamiento medial de agrupaciones de la sociedad civil y partidos políticos incidentes.

Fuente: Elaboración propia (2018).

Figura 3. Palabras más repetidas en la cobertura de la prensa digital

Se recogió en una nube de palabras, aquellas que más se repitieron en la cobertura del evento investigado. Resultando que sexo, ley, años, identidad, menores y proyecto, fueron las más usadas. 
Pía Ibarra Yáñez - Camila Amoyao Bravo - Eduardo Román Álvarez

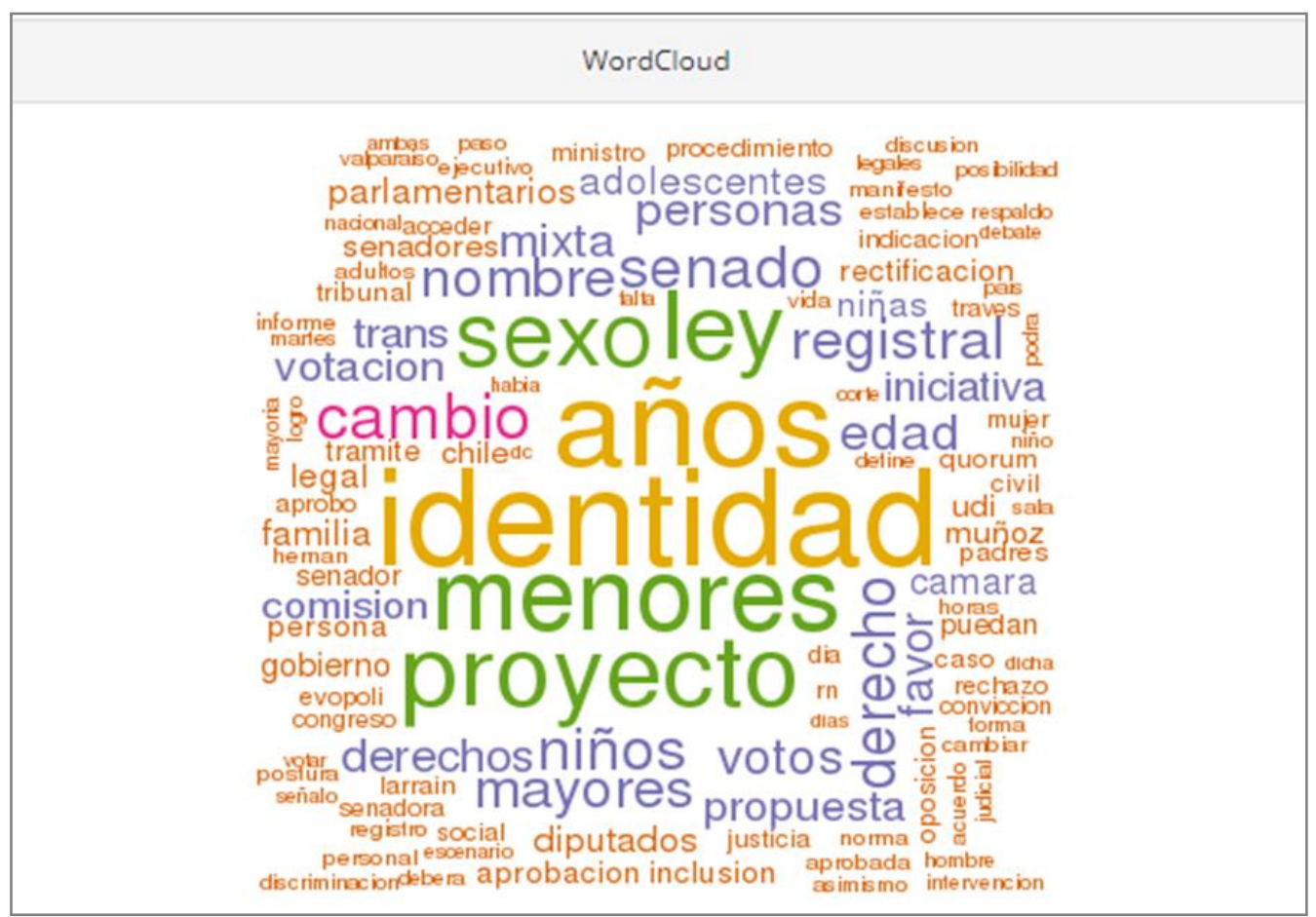

Figura 3. Palabras más repetidas en la cobertura de la prensa digital. Fuente: Elaboración propia (2018).

Figura 4. Cantidad de fuentes en promedio consultadas por los medios

El gráfico muestra el número de fuentes utilizadas en promedio por la totalidad de la muestra: Un 42\% tiende a consultar solo a una fuente, entre esos lidera Radio Agricultura, medio que menos fuentes consulta de forma reiterada; El Ciudadano; y el Líbero. Mientras que el 21,1\% consulta dos fuentes y el 18,4\% a tres. Pocos consultan a más de 3 fuentes, siendo La Tercera el medio que más registró. 
Análisis de la cobertura de la prensa digital chilena durante la discusión legislativa de la Ley de Identidad de Género en 2018

Pía Ibarra Yáñez - Camila Amoyao Bravo - Eduardo Román Álvarez

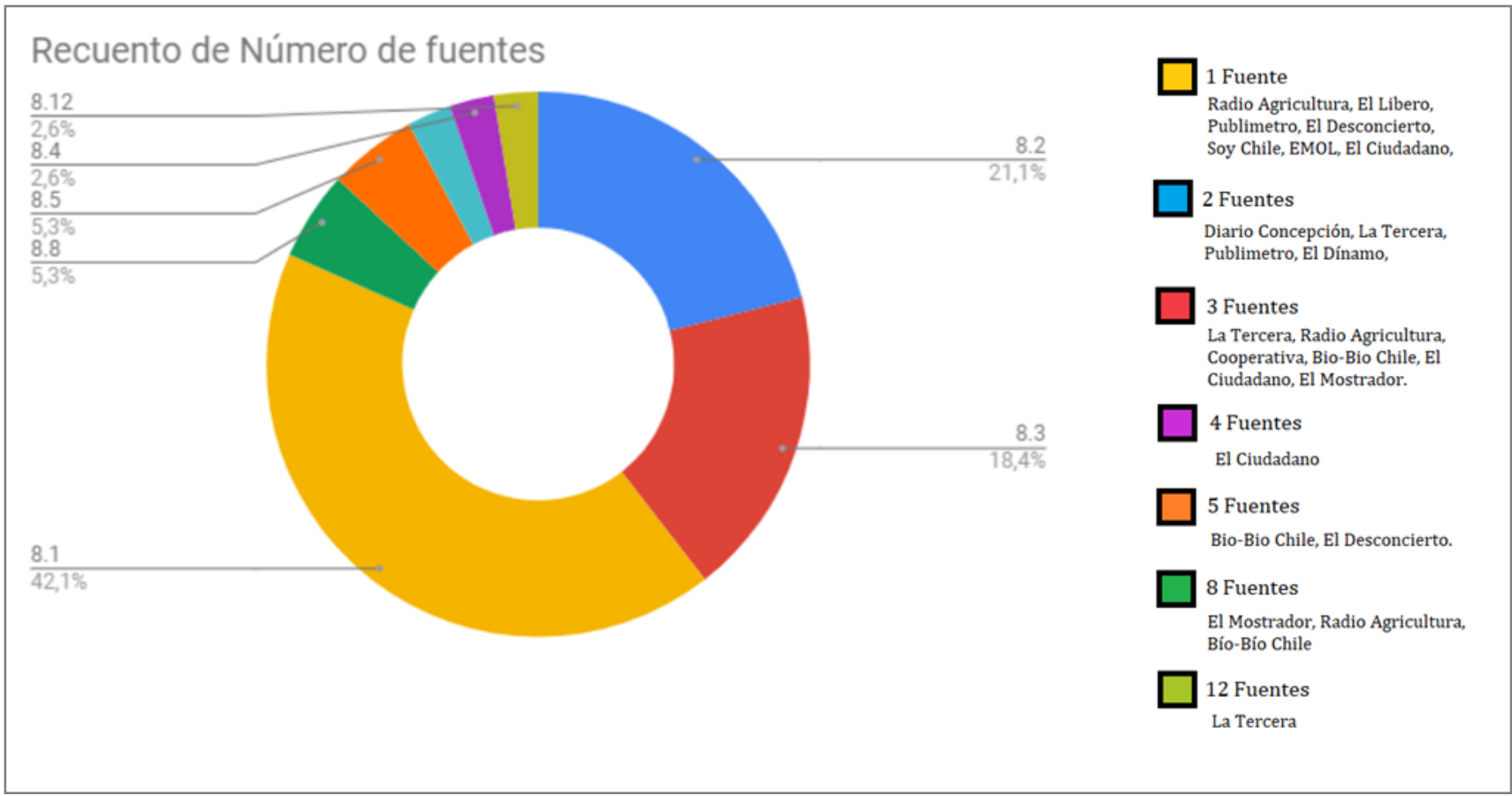

Figura 4. Cantidad de fuentes en promedio consultadas por los medios. Fuente: Elaboración propia (2018).

Figura 5. Recuento de temporalidad de la cobertura medial

La categoría muestra que durante los tres días anteriores a la votación en la Cámara Alta hubo una menor mediatización del tema, pero esta va en escalada a medida que se acerca la fecha de discusión final sobre la propuesta de la Comisión mixta en el Senado, la que después de aprobada va decantando con el transcurso de los días. Aun así, no baja su cobertura como en el primer día, ya que el ambiente continúa tensionado debido a que la ley aún no se publica. 
Análisis de la cobertura de la prensa digital chilena durante la discusión legislativa de la Ley de Identidad de Género en 2018

Pía Ibarra Yáñez - Camila Amoyao Bravo - Eduardo Román Álvarez

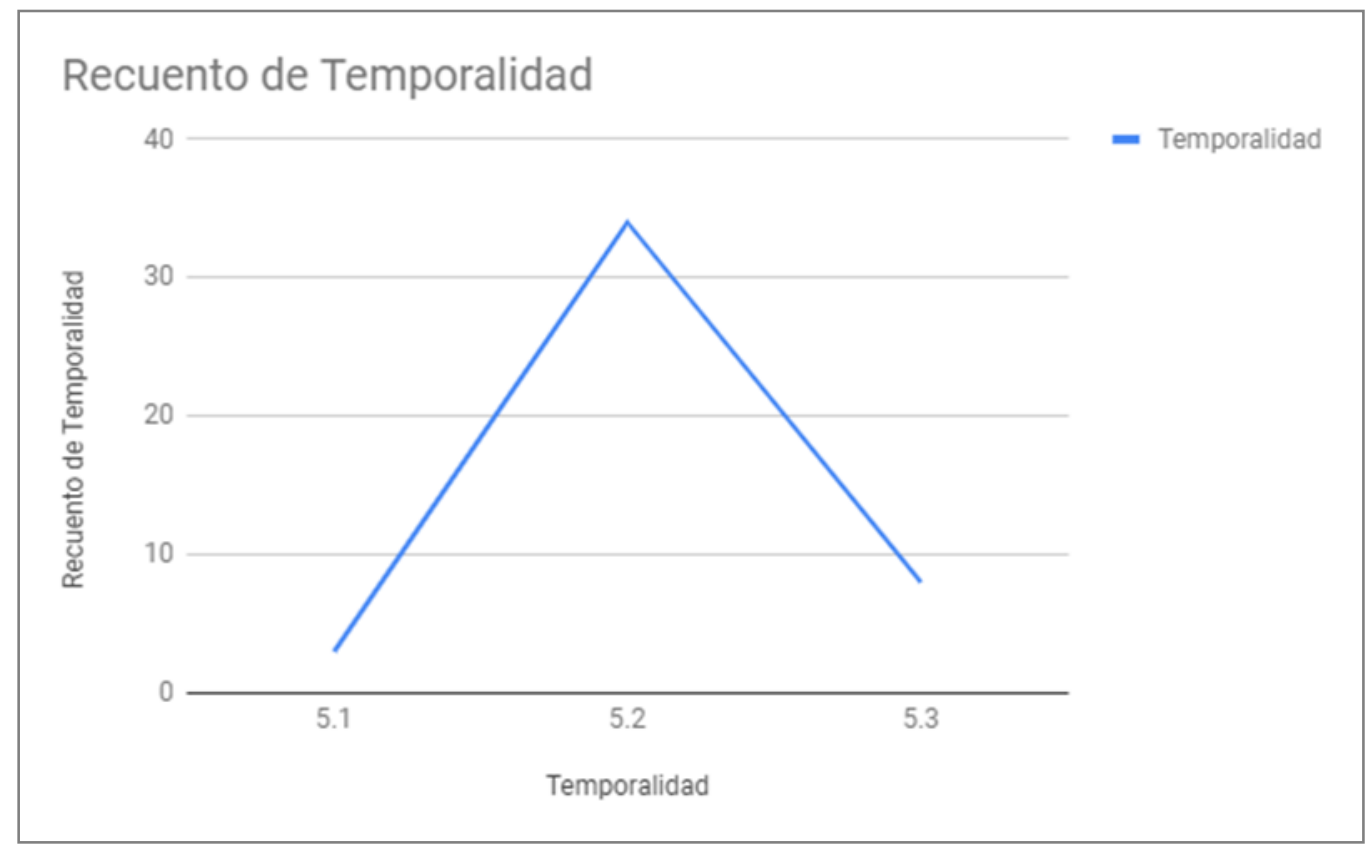

Figura 5. Recuento de temporalidad de la cobertura medial. Fuente: Elaboración propia (2018).

Figura 6. Tipos de fuentes consultadas

El gráfico evidencia que los tipos de fuentes que consultaron los medios respecto al tratamiento de la LIG fueron principalmente fuentes de gobierno (personeros de gobierno y oficialismo), las que constituyeron el grueso de las menciones que se identificaron. En tanto que fuentes del Poder Legislativo fueron las segundas que más registros anotaron. Otros (por ejemplo, miembros de comunidades religiosas como también de la diversidad sexual) ocupan el tercer nivel de las fuentes más consultadas. Cabe mencionar que se midieron tanto fuentes documentales como humanas. 
Análisis de la cobertura de la prensa digital chilena durante la discusión legislativa de la Ley de Identidad de Género en 2018

Pía Ibarra Yáñez - Camila Amoyao Bravo - Eduardo Román Álvarez

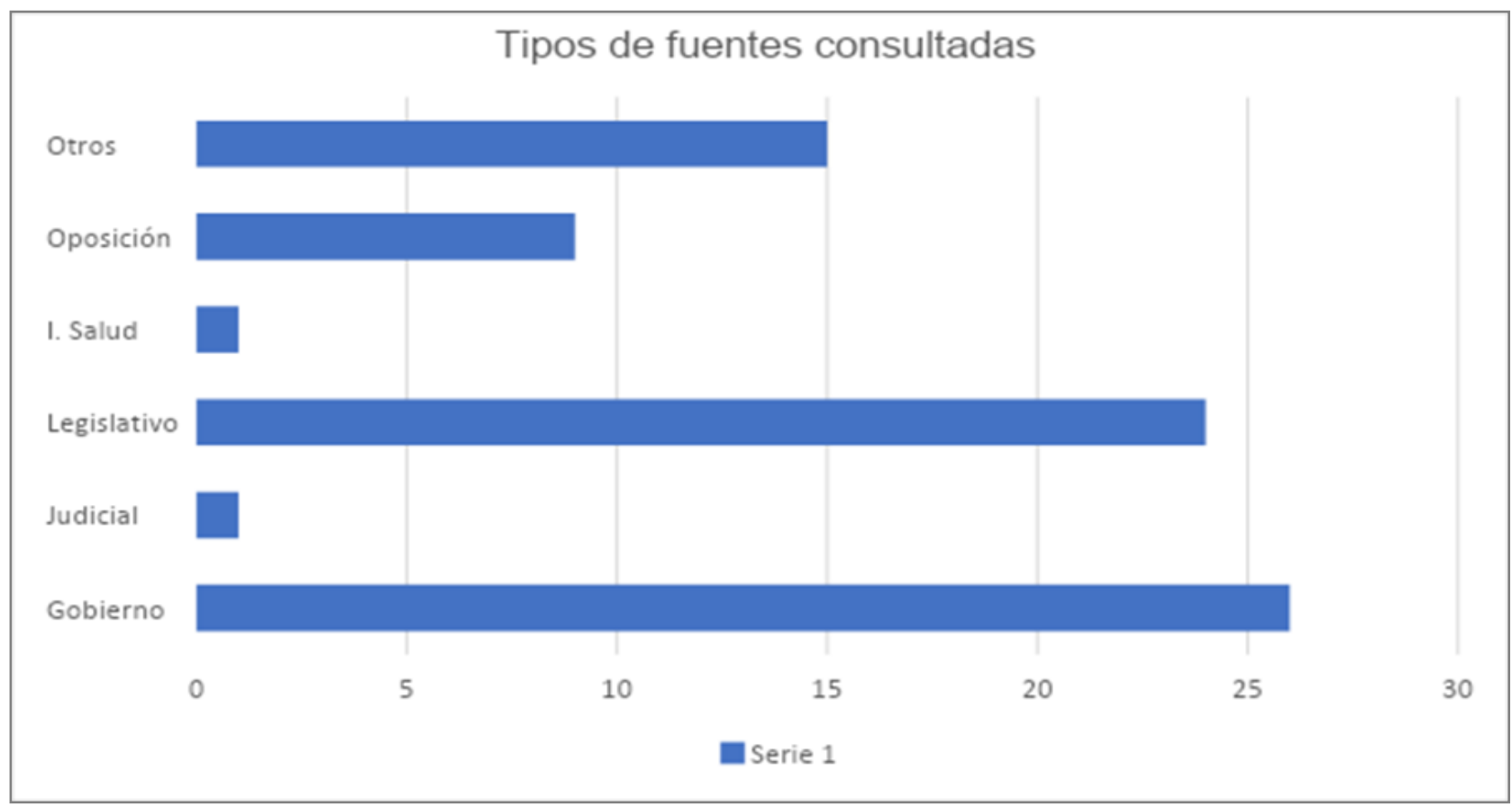

Figura 6. Tipos de fuentes consultadas. Fuente: Elaboración propia (2018).

Figura 7. Recuento de los actores políticos que aparecieron en la cobertura

El gráfico circular muestra a los actores políticos, quienes corresponden a los personajes que más se repiten en el tratamiento noticioso y que cumplen un rol dentro de este.

Con un 51,7\% los actores que más opinaron a través de la prensa fueron personeros de gobierno. Dentro de la derecha, si bien mayoritariamente se alineaban dentro del discurso que promovía el Ejecutivo, también existían diferencias (por ejemplo, Evópoli y miembros puntuales cercanos a esa administración tenían un discurso distinto).

El 7,3\% corresponde a actores identificados en este estudio como "Otros". Son quienes no pertenecen al sector político del país. Corresponden a personajes del mundo religioso (por ejemplo, evangélicos), miembros de la comunidad LGBTI, abogados, psicólogos, etc. 


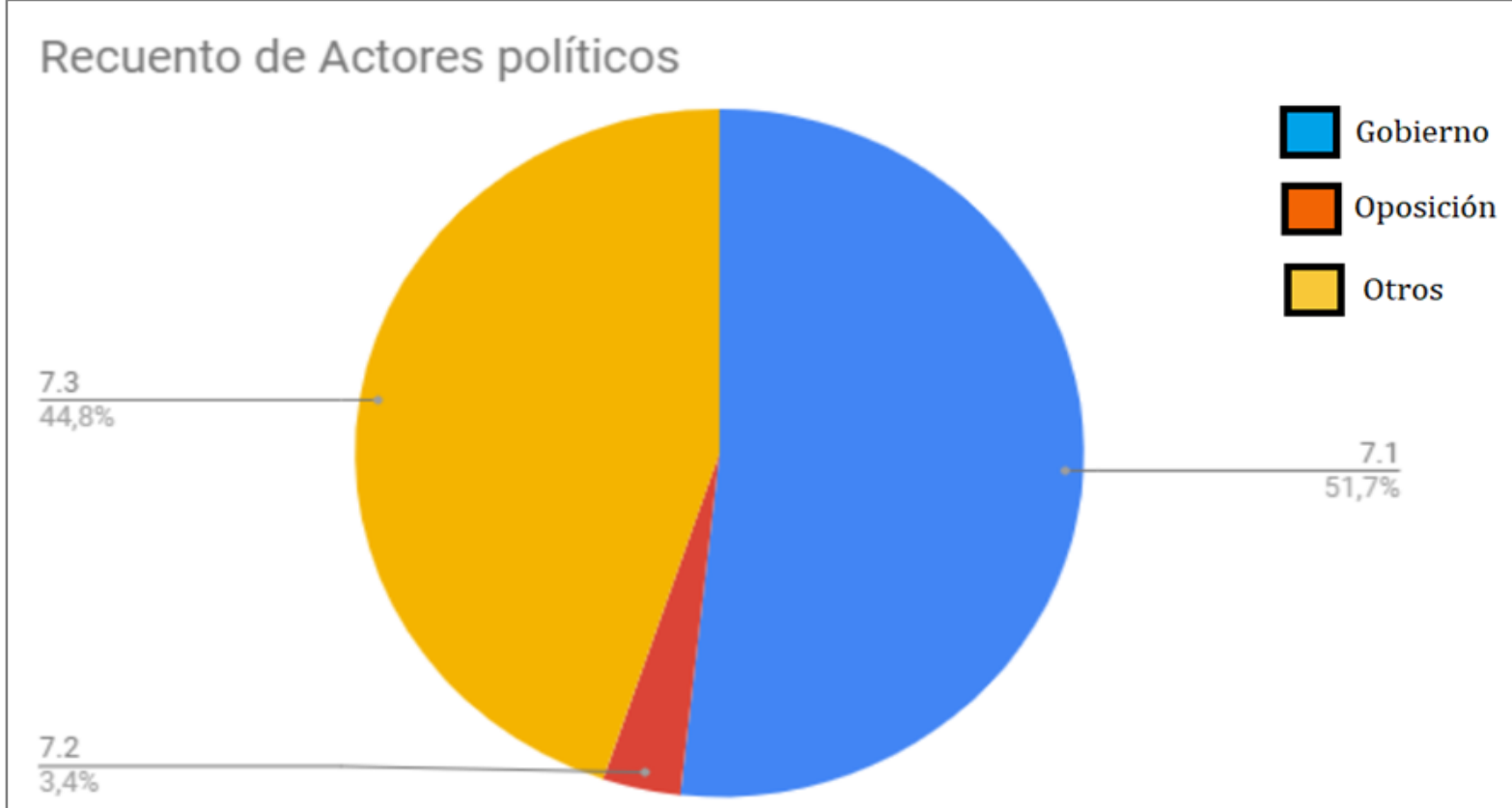

Figura 7. Recuento de los actores políticos que aparecieron en la cobertura. Fuente: Elaboración propia (2018).

\section{Conclusiones}

La prensa en general tiende a ser neutral pero sigue siéndolo por un estrecho margen, ya que hay una cantidad importante de informaciones que se inclinaron por una posición en contra a la implementación de la propuesta, mientras que la menor cantidad se enfocó en un tratamiento favorable a la ley, no obstante que las noticias difundidas se presentaron como una secuencia de "hechos".

Para diferenciar un "hecho" de un acontecimiento, los medios establecen un punto de referencia, puesto que lo que es un hecho para un sistema puede ser acontecimiento para otro. Ahora bien, sólo si el acontecimiento - y en ciertos casos intencionados, también el hecho- es difundido públicamente, se ha producido una noticia. Momento esencial en este proceso es el aislamiento lingüístico del acontecimiento, de modo que se pueda reducir como noticia.

Como los medios transmiten una cantidad determinada de noticias y descartan otras que, si se hubieran considerado dentro del tiempo o espacio definidos, también serían noticia, 
Pía Ibarra Yáñez - Camila Amoyao Bravo - Eduardo Román Álvarez

tanto la selección de los "hechos" acordes con determinada orientación ideológica como el modo de enfocarlas, no se hicieron al azar sino en virtud de la decisión de "alguien" que estimó que una ley inclusiva era al menos cuestionable o hasta repudiable.

Como lo que llega a ser noticia es parte de un proceso más largo y complejo de acciones que se prolongan en el tiempo, los "hechos" relatados son solo un destello de realidad que nos dan a conocer esas opciones y nos permiten entenderlas, ya que la estructura de los medios no permite transmitir acciones con duración en el tiempo, sólo "hechos" presentados como una suerte de instantánea entre pasado y futuro.

La noticia amplía el mercado cuando renuncia a ser instrumento descarado de proselitismo para aparecer separada de la opinión, pero las principales fuentes de informaciones se constituyen con interesados en difundir algunos hechos, expuestos desde una posición ideológica muy decidida, pero de apariencia neutral. Como la semantización está orientada a una apariencia de objetividad, el suministro de hechos también aparece como gratuito y el público acepta como regalo una propaganda, en este caso discriminadora, pero que aparenta estar protegiendo la integridad moral de la sociedad, ni más ni menos.

Es importante que nada de lo expuesto en los párrafos anteriores es casual ni espontáneo ni exclusivo respecto de una ley que se discutía en el parlamento, cuestionando un determinado orden social. El sistema político no sólo suministra hechos convertibles en noticias, sino que es una organización para producirlas. Sus agendas están trazadas en función de la publicidad que se obtiene con una actividad. En una misma edición, sin embargo, pueden coexistir hechos difundidos por grupos de intereses opuestos, porque —espontáneos o provocados- son necesidades técnicas de los medios.

El poco manejo de fuentes utilizadas muestra una deficiencia en la calidad de la información a la hora de emitirla, ya que estas son un recurso importante para la labor periodística porque son recursos imprescindibles para construir el relato y contar la noticia. También, para conferir credibilidad tanto a la información como al propio procedimiento usado para elaborarla. Esta carencia de fuentes hace que sea limitada en la realidad que da a conocer, inclinándose muchas veces por una postura o solo por describir lo que sucedió de manera macro, puesto que la profundización de un tema se mide según las fuentes consultadas. 
Pía Ibarra Yáñez - Camila Amoyao Bravo - Eduardo Román Álvarez

En cuanto a los actores políticos, el que aparezcan más personeros de gobierno indica que se está considerando su postura como predominante, generando que los discursos de los otros sectores tengan una menor cobertura, lo que decanta en una clara disparidad para el resto de quienes tienen una postura diferente a la del Ejecutivo, puesto que sus discursos tienen menos cabida y por lo tanto menor repercusión en la ciudadanía. Por otra parte, es importante recalcar que el lenguaje es un elemento de gran poder, porque tiene la facultad de extenderse en absolutamente todos los ámbitos de la vida. También, es esencial al momento de analizar los medios desde la temática del género, ya que muestra cómo estos van moldeando la realidad y construyendo una visión colectiva en la sociedad.

Finalmente, el artículo comprueba que la cobertura de prensa fue precaria, valiéndose de fuentes de información de partidos políticos más que de los grupos de interés afectados directamente. Demostrando de este modo que los medios de comunicación digital tienden a informar y seleccionar a sus fuentes según su línea editorial rozando sí la llamada neutralidad periodística.

\section{Bibliografía}

Adoni, H. y Mane, S. (1984). "Media and Social Construction of Reality: Toward an Integration of Theory and Research". Communication Research, 11(3).

https://doi.org/10.1177/009365084011003001

Alto Comisionado de las Naciones Unidas para los Refugiados. (2014). La protección de las personas LGBTI. Solicitudes de la condición de refugiado relacionadas con la orientación sexual y/o identidad de género. Recuperado de https://www.acnur.org/fileadmin/Documentos/Publicaciones/2014/9872.pdf

Braz, C. (2019). "Acá yo soy un pibe normal" - Narrativas sobre la espera y el acceso a derechos entre varones trans en Argentina. Sexualidad, Salud y Sociedad (Rio de Janeiro), (31), 119-138. https://doi.org/10.1590/1984-6487.sess.2019.31.07.a 
Análisis de la cobertura de la prensa digital chilena durante la discusión legislativa de la Ley de Identidad de Género en 2018

Pía Ibarra Yáñez - Camila Amoyao Bravo - Eduardo Román Álvarez

Del Río Reynaga, J. (1991). Teoría y Práctica de los Géneros Periodísticos Informativos. México: Editorial Diana.

Díez, J. (2013). "Explaining Policy Outcomes: The Adoption of Same-Sex Unions in Buenos Aires and Mexico City". Comparative Political Studies, 46(2), 212-235. https://doi.org/10.1177/0010414012453035

Fries, L. (2016). Diversidad sexual. En M. Amunátegui, J. Aylwin, C. Contreras, S. Donoso, C. Frontaura, R. Garretón, ... M. Núñez (Eds.), Manual de derechos humanos para comunicadores y comunicadoras (Eliana Largo ed., pp. 33-40). Recuperado de http://bibliotecadigital.indh.cl/handle/123456789/956

Gomiz, L. (1991). Teoría del Periodismo. Barcelona: Editorial Paidós.

Hall, S. (1981). La Cultura, los Medios de Comunicación y el Efecto Ideológico. México: Fondo de cultura económica.

Iguales. (s.f.). Incidencia política: Ley de identidad de Género. Recuperado de https://www.iguales.cl/incidencia-politica/ley-de-identidad-de-genero/

Ochoa Chaves, L., Castro Camacho, A., \& Cubero Hernández, E. (2017). "Leyes de comunicación en América Latina: derecho a la comunicación con perspectiva de género". Cuadernos Intercambio sobre Centroamérica y el Caribe, 14(2), 44.

https://doi.org/10.15517/c.a..v14i2.30942

Pájaro, J. (2016). "Del armario a los medios: significaciones imaginarias sobre la población LGBTI en la prensa de Cartagena de Indias, Colombia”. En Del armario a los medios: significaciones imaginarias sobre la población LGBTI en la prensa de Cartagena de Indias, Colombia (pp. 157-178). Recuperado de

https://rephip.unr.edu.ar/bitstream/handle/2133/18726/Del\%20armario\%20a\%201os\%20medios\% 20significaciones\%20imaginarias\%20sobre\%20la\%20poblaci\%C3\%B3n\%20LGBTI\%20en\%201 a\%20prensa $\% 20 \mathrm{de} \% 20 \mathrm{Cartagena} \% 20 \mathrm{de} \% 20$ Indias $\% 2 \mathrm{C} \% 20 \mathrm{Colombia}$.pdf? sequence=2\&isAllow $\mathrm{ed}=\mathrm{y}$

Programa de las Naciones Unidas para el Desarrollo. (2014). Sistematización del Proceso para la aprobación de la Ley de Identidad de Género en Argentina. Recuperado de https://es.scribd.com/document/235837311/Sistematizacion-Del-Proceso-de-La-Ley-deIdentidad-de-Genero-en-Argentina-Final

Quiteria, F. (2017, 18 agosto). América Latina avanza en el disfrute de derechos para personas trans | Amnistia Internacional | Venezuela. Recuperado 15 de diciembre de 2020, de https://www.amnistia.org/ve/blog/2017/08/3407/america-latina-avanza-en-el-disfrute-dederechos-para-personas-trans 
Análisis de la cobertura de la prensa digital chilena durante la discusión legislativa de la Ley de Identidad de Género en 2018

Pía Ibarra Yáñez - Camila Amoyao Bravo - Eduardo Román Álvarez

Rangel, D. (2015). "Extendiendo el derecho a todos los derechos: la difusión transnacional del reconocimiento legal de la identidad de género". Colombia Internacional, 87. http://dx.doi.org/10.7440/colombiaint87.2016.07

Rivadeneira, R. (1991). Periodismo: La Teoría General de los Sistemas y la Ciencia de las Comunicaciones. México: Editorial Trillas.

Rodrigo Alsina, M. (1993). La Construcción de la Noticia. Barcelona: Editorial Paidós.

Van Dijk, T. (1990). La Noticia como Discurso. Barcelona: Editorial Paidós.

Weeks, J. (2012). Lenguajes de la sexualidad. Buenos Aires, Argentina: Nueva Visión. 